\title{
A VARIATIONAL APPROACH TO THE YAU-TIAN-DONALDSON CONJECTURE
}

\author{
ROBERT BERMAN, SÉBASTIEN BOUCKSOM, AND MATTIAS JONSSON
}

\begin{abstract}
We give a new proof of a uniform version of the Yau-Tian-Donaldson conjecture for Fano manifolds with finite automorphism group, and of the semistable case of the conjecture. Our approach does not involve the continuity method or Cheeger-Colding-Tian theory. Instead, the proof is variational and uses pluripotential theory and certain non-Archimedean considerations.
\end{abstract}

\section{INTRODUCTION}

The goal of this note is to sketch a proof of the following version of the Yau-Tian-Donaldson conjecture in the Fano case.

Theorem A. Let $X$ be a Fano manifold with finite automorphism group. Then $X$ admits a KählerEinstein metric iff it is uniformly K-stable.

The notion of uniform K-stability used here was introduced in BHJ15a, Der14, following ideas originating in G. Székelyhidi's thesis (see also [Szé15]). It requires the Donaldson-Futaki invariant of any test configuration to be bounded below by a fixed positive multiple of a norm-like invariant ${ }^{1}$ detecting the non-triviality of a test configuration, see \&1. This notion, which makes sense for arbitrary polarized varieties, turns out to be equivalent to K-stability in the case of Fano manifolds, in view of CDS15] and the above result.

The main result in [CDS15] (see also [DS15, CSW15], and [Tia15]) is stronger than Theorem A since it allows $X$ to have nontrivial vector fields. It also uses K-(poly)stability instead of uniform K-stability. However, our proof avoids many of the subtle points in the previous approaches. For example, we do not use the continuity method, partial $C^{0}$-estimates, Cheeger-Colding-Tian theory, or the Kähler-Ricci flow.

Moreover, a variant of the proof of Theorem A gives the semistable version of the YTD conjecture:

Theorem B. Let $X$ be a Fano manifold. Then $X$ is K-semistable iff its greatest Ricci lower bound $R(X)$ is equal to 1 .

This was previously deduced from CDS15] in Li13. The right-hand condition means that, for any $\varepsilon>0, c_{1}(X)$ contains a Kähler metric $\omega_{\varepsilon}$ with $\operatorname{Ric}\left(\omega_{\varepsilon}\right) \geq(1-\varepsilon) \omega_{\varepsilon}$. By [Szé11], this amounts to the solvability of Aubin's continuity method up to any time $t<1$.

In order to describe our approach, recall that Kähler-Einstein metrics correspond to critical points of either the Mabuchi K-energy functional $M$ or the Ding functional $D$, both defined on the space $\mathcal{H}$ of Kähler potentials and which satisfy $D \leq M$. If $X$ is assumed to have finite automorphism group, known results Tia97, PSSW08 yield the equivalence between:

(i) $X$ has a Kähler-Einstein metric;

(ii) the Ding functional $D$ is coercive, i.e. $D \geq \delta J-C$ on $\mathcal{H}$ for some $\delta, C>0$;

(iii) the Mabuchi functional $M$ is coercive.

Date: September 16, 2015.

${ }^{1}$ One can show that the norm used is equivalent to the $L^{1}$-norm of a test configuration. 
Here $J \geq 0$ denotes the Aubin energy functional, a non-linear higher dimensional version of the classical Dirichlet functional. Note that (iii) $\Longrightarrow$ (i) can alternatively be established using a variational argument BBEGZ11, and that (i) $\Longrightarrow$ (ii) was recently given a very elegant proof in [DR15], based on new ideas that have had a strong influence on the strategy of proof of Theorem A proposed here.

Next, recall that a ray $\left(\phi_{t}\right)_{t \geq 0}$ in $\mathcal{H}$ corresponds to an $S^{1}$-invariant metric $\Phi$ on the pull-back of $-K_{X}$ to the product of $X$ with the punctured unit disc $\Delta^{*}$, the ray being called subgeodesic when $\Phi$ is plurisubharmonic (psh for short). Denoting by $F$ any of the functionals $M, D$ or $J$, the asymptotic behavior of $F\left(\phi_{t}\right)$ as $t \rightarrow+\infty$ is well-understood whenever the corresponding metric $\Phi$ extends to a smooth metric on a test configuration $(\mathcal{X}, \mathcal{L})$, i.e. a $\mathbf{C}^{*}$-equivariant partial compactification of the product of the polarized manifold $\left(X,-K_{X}\right)$ with $\mathbf{C}$. Indeed, we then have

$$
\lim _{t \rightarrow+\infty} \frac{F\left(\phi_{t}\right)}{t}=F^{\mathrm{NA}}(\mathcal{X}, \mathcal{L})
$$

where $F^{\mathrm{NA}}$ is the corresponding non-Archimedean functional introduced in BHJ15a]. For $F=D$, this is a reformulation of a key technical step in Berm15. For $F=M$ or $J$, this is established in BHJ15b, but closely related less precise formulations have appeared several times in the literature over the last two decades.

Denoting by $\operatorname{DF}(\mathcal{X}, \mathcal{L})$ the Donaldson-Futaki invariant of a (normal, ample) test configuration $(\mathcal{X}, \mathcal{L})$, we have $\operatorname{DF}(\mathcal{X}, \mathcal{L}) \geq M^{\mathrm{NA}}(\mathcal{X}, \mathcal{L}) \geq D^{\mathrm{NA}}(\mathcal{X}, \mathcal{L})$. Uniform K-stability is defined as the existence of $\delta>0$ such that $\mathrm{DF} \geq \delta J^{\mathrm{NA}}$, and turns out to be equivalent to $M^{\mathrm{NA}} \geq \delta J^{\mathrm{NA}}$ [BHJ15a].

Our approach to Theorem A consists in establishing the equivalence between (ii), (iii) above and

(ii') $D^{\mathrm{NA}} \geq \delta J^{\mathrm{NA}}$ for some $\delta>0$;

(iii') $M^{\mathrm{NA}} \geq \delta J^{\mathrm{NA}}$ for some $\delta>0$,

the implications (ii) $\Longrightarrow$ (ii') and (iii) $\Longrightarrow$ (iii') being immediate consequences of $(0.1)$.

In a first purely algebro-geometric step, we establish (iii') $\Longrightarrow$ (ii') (cf. Theorem 2.1 below), the converse implication being trivial since $M^{\mathrm{NA}} \geq D^{\mathrm{NA}}$. This is accomplished by using the Minimal Model Program, very much in the same way as in [LX14].

The heart of our proof is the implication (ii') $\Longrightarrow$ (iii). Arguing by contradiction, we assume $M$ is not coercive. Using a compactness argument inspired by Darvas and He DH14 (itself relying on the energy-entropy compactness theorem in (BBEGZ11]), we produce a subgeodesic ray along which $M$ has slow growth. As in DH14, this ray lies not in $\mathcal{H}$, but in the space $\mathcal{E}^{1}$ of metrics of finite energy, a space whose structure was recently clarified by Darvas [Dar14]. As in [DR15], to control the Mabuchi functional along the ray, we also use a recent result by Berndtsson and the first author [BB14] (see also [CLP14]) to the effect that $M$ is convex along geodesic segments.

Since the Ding functional $D$ is dominated by the Mabuchi functional, $D$ also has slow growth along the geodesic ray. If $\Phi$ happens to extend to a bounded metric on some test configuration $(\mathcal{X}, \mathcal{L})$ of $\left(X,-K_{X}\right)$, the slope of $D$ at infinity is given by $D^{\mathrm{NA}}(\mathcal{X}, \mathcal{L})$, and (ii') yields a contradiction. In the general case, we may assume that $\Phi$ extends to a psh metric on the pullback of $-K_{X}$ to $X \times \Delta$, but the singularities along the central fiber may be quite complicated. Nevertheless, the slope of $D$ at infinity can be analyzed using the multiplier ideals of $m \Phi, m \in \mathbf{Z}_{\geq 0}$; these give rise to a sequence of test configurations to which we can apply the assumption (ii') and derive a contradiction. This step is quite subtle and involves some non-Archimedean analysis in the spirit of [BFJ08, BFJ12] in order to calculate the slope at infinity of the Ding functional.

This note is rather brief. Details and further results will appear elsewhere. In particular, the method can most likely be extended to treat the case of varieties with log terminal singularities.

Acknowledgment. We would like to thank Tamás Darvas, Ruadhaí Dervan, Tomoyuki Hisamoto, Julius Ross, Yanir Rubinstein, Song Sun, David Witt Nyström and, especially, Gábor Székelyhidi for useful comments. 


\section{Preliminaries}

Let $X$ be a Fano manifold of dimension $n$. Set $L:=-K_{X}$ and $V=\left(c_{1}(L)^{n}\right)$. We use additive notation for metrics. Let $\mathcal{H}$ be the space of smooth, strictly psh metrics on $L$. Fix a reference metric $\phi_{0} \in \mathcal{H}$.

1.1. Functionals on $\mathcal{H}$. For details on the following, see for instance BBGZ13, BBEGZ11. The (Monge-Ampère, or Aubin-Mabuchi) energy of $\phi$ is

$$
E(\phi)=\frac{1}{(n+1) V} \sum_{j=0}^{n} \int_{X}\left(\phi-\phi_{0}\right)\left(d d^{c} \phi\right)^{j} \wedge\left(d d^{c} \phi_{0}\right)^{n-j} .
$$

The $J$-energy is

$$
J(\phi)=V^{-1} \int\left(\phi-\phi_{0}\right)\left(d d^{c} \phi_{0}\right)^{n}-E(\phi) \geq 0 .
$$

In the current Fano setting, the Ding functional is defined by $D=L-E$, where

$$
L(\phi)=-\frac{1}{2} \log \int e^{-2 \phi}
$$

with $e^{-2 \phi}$ symbolically denoting the volume form canonically attached to the metric $\phi$, and the Mabuchi functional becomes $M=H-E^{*}$, where

$$
E^{*}(\phi)=V^{-1} \int\left(\phi-\phi_{0}\right)\left(d d^{c} \phi\right)^{n}-E(\phi) \geq 0
$$

is the pluricomplex energy of the probability measure $V^{-1}\left(d d^{c} \phi\right)^{n}$, and $H(\phi) \geq 0$ is its entropy with respect to the reference measure $V^{-1}\left(d d^{c} \phi_{0}\right)^{n}$. We have $C^{-1} J \leq E^{*} \leq C J$ for some constant $C>0$.

All these functionals extend to the space $\mathcal{E}^{1}$ of metrics of finite energy, with $H$ (and hence $M$ ) possibly infinite valued.

1.2. Valuations, non-Archimedean metrics, and test configurations BHJ15a. A (real rank 1) valuation $v$ on (the function field of) $X$ is divisorial if $v=c \operatorname{ord}_{E}$, where $c>0$ and $E$ is a prime divisor on a normal variety $Y$ mapping birationally to $X$. The $\log$ discrepancy of $v$ is then $A_{X}(v)=c\left(1+\operatorname{ord}_{E}\left(K_{Y / X}\right)\right)$, where $K_{Y / X}$ is the relative canonical divisor. The projection $X \times \mathbf{C} \rightarrow X$ takes divisorial valuations to divisorial (or trivial) valuations. Further, every divisorial valuation on $X$ has a unique preimage $w$ on $X \times \mathbf{C}$ that is $\mathbf{C}^{*}$-invariant (under the action on the second factor) satisfies $v(\tau)=1$, with $\tau$ denoting the coordinate on $\mathbf{C}$; then $A_{X \times \mathbf{C}}(w)=A_{X}(v)+1$.

A non-Archimedean metric on $L$ will be viewed as a certain type of function $\varphi$ on the Berkovich analytification of $X$ with respect to the trivial norm on $\mathbf{C}$. In particular, $\varphi$ restricts to a function on the space of divisorial valuations on $X$, and is in fact uniquely determined by this restriction.

Every normal test configuration $(\mathcal{X}, \mathcal{L})$ of $(X, L)$ defines a non-Archimedean metric $\varphi=\varphi_{\mathcal{X}, \mathcal{L}}$ as follows. Pick a normal variety $\mathcal{Y}$ with $\mathbf{C}^{*}$-equivariant birational morphisms $\pi: \mathcal{Y} \rightarrow X \times \mathbf{C}$ and $\rho: \mathcal{Y} \rightarrow \mathcal{X}$, and let $p_{1}: X \times \mathbf{C} \rightarrow X$ be the projection. Then $\rho^{*} \mathcal{L}-\pi^{*} p_{1}^{*} L=\mathcal{O}_{\mathcal{Y}}(D)$ for a unique Q-divisor $D$ supported on the central fiber. We have $\varphi\left(c \operatorname{ord}_{E}\right)=c \operatorname{ord}_{E}(D)$ for every irreducible component $E$ of the central fiber of $\mathcal{Y}$. Varying $\mathcal{Y}$ determines $\varphi$ completely.

Write $\mathcal{H}^{\mathrm{NA}}$ for the set of non-Archimedean metrics defined by normal (semi)ample test configurations.

1.3. Non-Archimedean functionals. In [BHJ15a, natural non-Archimedean versions $E^{\mathrm{NA}}, J^{\mathrm{NA}}$, and $M^{\mathrm{NA}}$ of the basic functionals on $\mathcal{H}$ are introduced. They are defined as functionals $\mathcal{H}^{\mathrm{NA}} \rightarrow \mathbf{R}$, with

$$
J^{\mathrm{NA}}(\varphi)=\sup \varphi-E^{\mathrm{NA}}(\varphi)
$$


for all $\varphi \in \mathcal{H}^{\mathrm{NA}}$. The functional $M^{\mathrm{NA}}$ is closely related (but not equal) to the Donaldson-Futaki invariant DF. More precisely, we have

$$
M^{\mathrm{NA}}(\mathcal{X}, \mathcal{L})=\operatorname{DF}(\mathcal{X}, \mathcal{L})+V^{-1}\left(\left(\mathcal{X}_{0, \text { red }}-\mathcal{X}_{0}\right) \cdot \mathcal{L}^{n}\right)
$$

so that $M^{\mathrm{NA}}(\mathcal{X}, \mathcal{L}) \leq \operatorname{DF}(\mathcal{X}, \mathcal{L})$ with equality when $\mathcal{X}_{0}$ is reduced. Uniform $\mathrm{K}$-stability is equivalent to an estimate $M^{\mathrm{NA}} \geq \delta J^{\mathrm{NA}}$ on $\mathcal{H}^{\mathrm{NA}}$.

In addition, let us introduce $L^{\mathrm{NA}}$ and $D^{\mathrm{NA}}:=L^{\mathrm{NA}}-E^{\mathrm{NA}}$ by the formula

$$
L^{\mathrm{NA}}(\varphi)=\inf _{v}\left(A_{X}(v)+\varphi(v)\right)
$$

where $v$ ranges over divisorial valuations on $X$ (compare [Berm15, Proposition 3.8]). We note that

$$
D^{\mathrm{NA}} \leq M^{\mathrm{NA}} \text { and } D^{\mathrm{NA}} \leq J^{\mathrm{NA}} .
$$

We say that $X$ is uniformly Ding-stable if $D^{\mathrm{NA}} \geq \delta J^{\mathrm{NA}}$ on $\mathcal{H}^{\mathrm{NA}}$ for some $\delta>0$. This trivially implies uniform K-stability, and the converse is also true, as we shall see in Theorem 2.1 .

\section{From uniform K-Stability to Uniform Ding Stability}

As a first step towards the proof of Theorem A, we show:

Theorem 2.1. Let $X$ be a smooth Fano manifold and $\delta \geq 0$. Then $M^{\mathrm{NA}} \geq \delta J^{\mathrm{NA}}$ holds on $\mathcal{H}^{\mathrm{NA}}$ iff $D^{\mathrm{NA}} \geq \delta J^{\mathrm{NA}}$ on $\mathcal{H}^{\mathrm{NA}}$

Proof. One direction is clear since $M^{\mathrm{NA}} \geq D^{\mathrm{NA}}$. Conversely, suppose $M^{\mathrm{NA}} \geq \delta J^{\mathrm{NA}}$ on $\mathcal{H}^{\mathrm{NA}}$. We first observe that $D^{\mathrm{NA}}$ and $M^{\mathrm{NA}}$ coincide on any test configuration $(\mathcal{X}, \mathcal{L})$ which is lc (in the sense that the pair $\left(\mathcal{X}, \mathcal{X}_{0}\right)$ has log canonical singularities) and such that $\mathcal{L} \equiv-K_{\mathcal{X}}$ (numerical equivalence).

In order to establish $D^{\mathrm{NA}}(\mathcal{X}, \mathcal{L}) \geq \delta J^{\mathrm{NA}}(\mathcal{X}, \mathcal{L})$ for an arbitrary normal, semiample test configuration, we may assume wlog that $(\mathcal{X}, \mathcal{L})$ is semistable (and hence lc), since both sides are homogeneous under finite base change. Following the procedure of [LX14, we then use the Minimal Model Program to produce a continuous path $\left(\varphi_{t}\right)_{t \in[0, T]}$ in $\mathcal{H}^{\mathrm{NA}}$ with $\varphi_{0}=\varphi_{(\mathcal{X}, \mathcal{L})}, \varphi_{T}$ associated to an lc test configuration $\left(\mathcal{X}^{\prime}, \mathcal{L}^{\prime}\right)$ with $\mathcal{L}^{\prime} \equiv-K_{\mathcal{X}^{\prime}}$, and such that $t \mapsto D^{\mathrm{NA}}\left(\varphi_{t}\right) / J^{\mathrm{NA}}\left(\varphi_{t}\right)$ is non-increasing. We will then get as desired

$$
\frac{D^{\mathrm{NA}}(\varphi)}{J^{\mathrm{NA}}(\varphi)} \geq \frac{D^{\mathrm{NA}}\left(\varphi_{T}\right)}{J^{\mathrm{NA}}\left(\varphi_{T}\right)}=\frac{M^{\mathrm{NA}}\left(\varphi_{T}\right)}{J^{\mathrm{NA}}\left(\varphi_{T}\right)} \geq \delta
$$

More precisely, we run an MMP with scaling by $\mathcal{H}:=r \mathcal{L}-K_{\mathcal{X}}, r \gg 1$, as in [LX14, $\left.\S 5.1\right]$. This yields a sequence of rational numbers $1=: \lambda_{0}>\cdots>\lambda_{k}>\lambda_{k+1}:=\frac{1}{r+1}$, a sequence of $\mathbf{C}^{*}$-equivariant divisorial contractions and flips

$$
\mathcal{X}=: \mathcal{X}^{0} \rightarrow \mathcal{X}^{1} \rightarrow-\rightarrow-\rightarrow \mathcal{X}^{k}
$$

and, for each $j$, an affine path of lc, semiample test configurations $\left(\mathcal{X}^{j}, \mathcal{L}_{\lambda}^{j}\right), \lambda \in\left[\lambda_{j+1}, \lambda_{j}\right]$, such that

$$
K_{\mathcal{X}^{j}}+\mathcal{L}_{\lambda}^{j}=\frac{r \lambda}{(r+1) \lambda-1}\left(K_{\mathcal{X}^{j}}+\mathcal{L}^{j}\right)
$$

with $\mathcal{L}^{j}$ the push-forward of $\mathcal{L}$ to $\mathcal{X}^{j}$. Reparametrizing by $e^{t}=\frac{r \lambda}{(r+1) \lambda-1}$ then yields the desired path $\left(\varphi_{t}\right)$ in $\mathcal{H}^{\mathrm{NA}}$. Indeed using 2.1 we prove that

$$
\frac{d}{d t}\left(D^{\mathrm{NA}}\left(\varphi_{t}\right)-J^{\mathrm{NA}}\left(\varphi_{t}\right)\right)=D^{\mathrm{NA}}\left(\varphi_{t}\right)-J^{\mathrm{NA}}\left(\varphi_{t}\right) \leq 0
$$

and

It follows that

$$
\frac{d}{d t} J^{\mathrm{NA}}\left(\varphi_{t}\right)=J^{\mathrm{NA}}\left(\varphi_{t}\right)-M^{\mathrm{NA}}\left(\varphi_{t}\right)
$$

$$
\left(\frac{d}{d t} D^{\mathrm{NA}}\left(\varphi_{t}\right)\right) J^{\mathrm{NA}}\left(\varphi_{t}\right)-D^{\mathrm{NA}}\left(\varphi_{t}\right)\left(\frac{d}{d t} J^{\mathrm{NA}}\left(\varphi_{t}\right)\right)=\left(D^{\mathrm{NA}}\left(\varphi_{t}\right)-J^{\mathrm{NA}}\left(\varphi_{t}\right)\right) M^{\mathrm{NA}}\left(\varphi_{t}\right) \leq 0,
$$


since $M^{\mathrm{NA}} \geq \delta J^{\mathrm{NA}} \geq 0$ by assumption, and we conclude as desired that $D^{\mathrm{NA}} / J^{\mathrm{NA}}$ is non-increasing along the path $\left(\varphi_{t}\right)$.

Remark 2.2. The path $\left(\varphi_{t}\right)$ can be seen as a non-Archimedean version of the Kähler-Ricci flow.

Remark 2.3. The condition $M^{\mathrm{NA}} \geq 0$ is equivalent to $K$-semistability, $c f$. [BHJ15a]. The case $\delta=0$ of Theorem 2.1 therefore says that K-semistability is also equivalent to Ding semistability, thereby showing that the main result of Fuj15 is also valid assuming only $X$ to be K-semistable.

\section{From uniform Ding stability to coercivity of the K-energy}

Suppose $D^{\mathrm{NA}} \geq \delta J^{\mathrm{NA}}$ on $\mathcal{H}^{\mathrm{NA}}$, where $\delta \in(0,1)$, and pick $\delta^{\prime} \in(0, \delta)$. We shall show that $M \geq \delta^{\prime} J-C$ on $\mathcal{H}$ for some constant $C>0$. As explained in the introduction, this proves Theorem A.

Arguing by contradiction, we assume there exists a sequence $\left(\phi_{j}\right)_{1}^{\infty}$ in $\mathcal{H}$ such that

$$
M\left(\phi_{j}\right) \leq \delta^{\prime} J\left(\phi_{j}\right)-j
$$

Since $M$ and $J$ are translation invariant, we may assume that $\phi_{j}$ is normalized so that $\sup \left(\phi_{j}-\phi_{0}\right)=$ 0 . The inequality $M \geq-n J$, which always holds, then implies $J\left(\phi_{j}\right) \geq \frac{j}{n+\delta^{\prime}} \rightarrow+\infty$, and hence $E\left(\phi_{j}\right) \leq-J\left(\phi_{j}\right) \rightarrow-\infty$.

3.1. Step 1: construction of a geodesic ray in $\mathcal{E}^{1}$. We largely follow Darvas and He [DH14]. Denote by $d_{1}$ the distance introduced by Darvas [Dar14] on the space $\mathcal{E}^{1}$ of finite energy, so that $d_{1}$ defines the topology of convergence in energy.

For each $j$ we let $\left(\phi_{j, t}\right)_{0 \leq t \leq T_{j}}$ be the geodesic segment connecting $\phi_{0}$ to $\phi_{j}$, parametrized so that $T_{j}=-E\left(\phi_{j}\right)$. By [BBGZ13, Proposition 6.2] and [Dar13, Theorem 1] we have $E\left(\phi_{j, t}\right)=-t$ and $\sup \left(\phi_{j, t}-\phi_{0}\right)=0$, respectively. Thus $J\left(\phi_{j, t}\right) \leq \sup \left(\phi_{j, t}-\phi_{0}\right)-E\left(\phi_{j, t}\right)=t$. In particular, $J\left(\phi_{j}\right) \leq T_{j}$, so $M\left(\phi_{j}\right) \leq \delta^{\prime} T_{j}-j<\delta^{\prime} T_{j}$ for all $j$. By [BB14], $M$ is convex along geodesic segments, so $M\left(\phi_{j, t}\right) \leq \frac{t}{T_{j}} M\left(\phi_{j}\right) \leq \delta^{\prime} t$ for $t \leq T_{j}$. Since $M \geq H-n J$, this yields $H\left(\phi_{j, t}\right) \leq\left(\delta^{\prime}+n\right) t$ for $t \leq T_{j}$, so for fixed $T>0$ and $t \leq T$, the metrics $\phi_{j, t}$ lie in the set

$$
\mathcal{K}_{T}:=\left\{\phi \in \mathcal{E}^{1} \mid \sup \left(\phi-\phi_{0}\right)=0 \text { and } H(\phi) \leq\left(\delta^{\prime}+n\right) T\right\},
$$

which is a compact subset of the metric space $\left(\mathcal{E}^{1}, d_{1}\right)$ by [BBEGZ11. By the geodesic property, we also have

$$
d_{1}\left(\phi_{j, t}, \phi_{j, s}\right)=d_{1}\left(\phi_{j, 1}, \phi_{0}\right)|t-s| \leq C\left(J\left(\phi_{j, 1}\right)+1\right)|t-s| \leq 2 C|t-s|,
$$

which shows that $t \mapsto \phi_{j, t}$ restrict to uniformly Lipschitz continuous maps $[0, T] \rightarrow \mathcal{K}_{T}$. Combining Ascoli's Theorem with a diagonal argument we may assume, after passing to a subsequence, that $\phi_{j, t}$ converges to a geodesic ray $\left(\phi_{t}\right)_{t \geq 0}$ in $\mathcal{E}^{1}$, uniformly for each compact time interval. Note in particular that $E\left(\phi_{t}\right)=-t$ for all $t$.

The geodesic ray $\left(\phi_{t}\right)$ defines an $S^{1}$-invariant metric $\Phi$ on $p_{1}^{*} L$ over $X \times \Delta^{*}$, such that the restriction of $\Phi$ to $X \times\{\tau\}$ is equal to $\phi_{\log |\tau|^{-1}}$. We claim that $\Phi$ is psh. To see this, note that the geodesic segment $\left(\phi_{j, t}\right)$ defines an $S^{1}$-invariant psh metric $\Phi_{j}$ on $p_{1}^{*} L$ over $X \times A_{j}$, where $A_{j}=\left\{e^{-T_{j}}<\right.$ $|\tau|<1\} \subset \mathbf{C}$. The locally uniform convergence of $\phi_{j, t}$ to $\phi_{t}$ implies that $\Phi_{j}$ converges to $\Phi$ locally uniformly in the $L^{1}$-topology; hence $\Phi$ is psh.

Now $M$ is lsc on $\mathcal{K}_{T}$, so $M\left(\phi_{t}\right) \leq \liminf _{j} M\left(\phi_{j, t}\right) \leq \delta^{\prime} t$. Hence $D\left(\phi_{t}\right) \leq M\left(\phi_{t}\right) \leq \delta^{\prime} t$ for $t \geq 0$.

3.2. Step 2: Approximation by test configurations. Since $\sup \left(\phi_{t}-\phi_{0}\right)=0$ for all $t, \Phi$ extends to a psh metric on $p_{1}^{*} L$ over $X \times \Delta$. It has zero Lelong numbers at all points on $X \times \Delta^{*}$ since $\phi_{t} \in \mathcal{E}^{1}$ for all $t$. The generic Lelong number along $X \times\{0\}$ is also zero. As a consequence, the multiplier ideals $\mathcal{J}(m \Phi)$ are cosupported on proper subvarieties of the central fiber $X \times\{0\}$, and they are of course $\mathbf{C}^{*}$-invariant.

We claim that there exists $m_{0}$ large enough such that the sheaf $\mathcal{O}\left(\left(m+m_{0}\right) p_{1}^{*} L\right) \otimes \mathcal{J}(m \Phi)$ is generated by its global sections on $X \times \Delta$ for each $m \geq 1$. More precisely, given a very ample line 
bundle $H$ on $X$, it is enough to choose $m_{0}$ such that $A:=m_{0} L-K_{X}-(n+1) H$ is ample on $X$. Indeed, as first observed in [DEL00, Corollary 1.5], this is a consequence of the Nadel vanishing theorem and the Castelnuovo-Mumford criterion for global generation. More precisely, since $\Delta$ is Stein, it is enough to show that $\mathcal{O}\left(\left(m+m_{0}\right) p_{1}^{*} L\right) \otimes \mathcal{J}(m \Phi)$ is $p_{2}$-globally generated, with $p_{2}: X \times \Delta \rightarrow \Delta$ denoting the second projection. By the relative version of the Castelnuovo-Mumford criterion, this will be the case as soon as

$$
R^{j}\left(p_{2}\right)_{*}\left(\mathcal{O}\left(\left(m+m_{0}\right) p_{1}^{*} L-j p_{1}^{*} H\right) \otimes \mathcal{J}(m \Phi)\right)=0
$$

for all $j \geq 1$, which is a consequence of Nadel's vanishing (compare [BFJ12, Theorem B.8]).

Denote by $\mu_{m}: \mathcal{X}_{m} \rightarrow X \times \mathbf{C}$ the normalized blow-up of $X \times \mathbf{C}$ along $\mathcal{J}(m \Phi)$, with exceptional divisor $E_{m}$, and set $\mathcal{L}_{m}:=\mu_{m}^{*} p_{1}^{*} L-\frac{1}{m+m_{0}} E_{m}$. Then $\left(\mathcal{X}_{m}, \mathcal{L}_{m}\right)$ is a normal, semiample test configuration for $(X, L)$, inducing a non-Archimedean metric $\varphi_{m} \in \mathcal{H}^{\mathrm{NA}}$ with $\sup \varphi_{m}=0$, given by

$$
\varphi_{m}(v)=\frac{1}{m+m_{0}} v(\mathcal{J}(m \Phi))
$$

for each $\mathbf{C}^{*}$-invariant divisorial valuation $v$ on $X \times \mathbf{C}$.

For each $m \geq 1$, pick any $S^{1}$-invariant smooth psh metric $\Psi_{m}$ on the Q-line bundle $\mathcal{L}_{m}$. By the results of Berm15, BHJ15b mentioned in the introduction, the corresponding subgeodesic ray $\left(\phi_{m, t}\right)$ satisfies

$$
\lim _{t \rightarrow+\infty} \frac{1}{t} L\left(\phi_{m, t}\right)=L^{\mathrm{NA}}\left(\varphi_{m}\right) \text { and } \lim _{t \rightarrow+\infty} \frac{1}{t} E\left(\phi_{m, t}\right)=E^{\mathrm{NA}}\left(\varphi_{m}\right)=-J^{\mathrm{NA}}\left(\varphi_{m}\right) .
$$

The psh metric $\Phi_{m}$ on $p_{1}^{*} L$ over $X \times \mathbf{C}$ induced by $\Psi_{m}$ has analytic singularities of type $\mathcal{J}(m \Phi)^{1 /\left(m+m_{0}\right)}$. As a consequence of Demailly's local regularization theorem [Dem92, Proposition 3.1], $\Phi_{m}$ is therefore less singular than $\Phi$. More precisely, for each $r<1$, we can find a constant $C_{r, m}>0$ such that $\Phi_{m} \geq \Phi-C_{m, r}$ over $X \times \Delta_{r}$. By monotonicity of $E$, the corresponding subgeodesic rays satisfy

$$
E\left(\phi_{m, t}\right) \geq E\left(\phi_{t}\right)-C_{m, r}=-t-C_{m, r}
$$

for $t>-\log r$, and we infer

$$
E^{\mathrm{NA}}\left(\varphi_{m}\right)=\lim _{t \rightarrow \infty} \frac{1}{t} E\left(\phi_{m, t}\right) \geq-1
$$

3.3. Step 3: asymptotics of $L$ along the geodesic ray. We now relate the slope at infinity of $L\left(\phi_{t}\right)$ to the asymptotics of the non-Archimedean functional $L^{\mathrm{NA}}\left(\varphi_{m}\right)$ as $m \rightarrow \infty$. We shall prove that

$$
\lim _{m \rightarrow+\infty} L^{\mathrm{NA}}\left(\varphi_{m}\right)=\lim _{t \rightarrow+\infty} \frac{1}{t} L\left(\phi_{t}\right) .
$$

Theorem 3.1. Let $\left(\phi_{t}\right)$ be a subgeodesic ray in $\mathcal{E}^{1}$ normalized by $\sup \left(\phi_{t}-\phi_{0}\right)=0$, and let $\Phi$ be the corresponding $S^{1}$-invariant psh metric on the pull-back of $-K_{X}$ to $X \times \Delta$. Then:

$$
\lim _{t \rightarrow+\infty} \frac{1}{t} L\left(\phi_{t}\right)=\inf _{w}\left(A_{X \times \mathbf{C}}(w)-1-w(\Phi)\right),
$$

where $w$ ranges over $\mathbf{C}^{*}$-invariant divisorial valuations on $X \times \mathbf{C}$ such that $w(\tau)=1$, and $A_{X \times \mathbf{C}}(w)$ is the log discrepancy of such a valuation.

Here $w(\Phi)$ is to be interpreted as a generic Lelong number on a suitable blowup, see [BFJ08].

Let us deduce 3.2 from Theorem 3.1. By BHJ15a], the projection map $X \times \mathbf{C} \rightarrow X$ induces a bijection between $\mathbf{C}^{*}$-invariant divisorial valuations $w$ on $X \times \mathbf{C}$ satisfying $w(\tau)=1$ and divisorial (or trivial) valuations $v$ on $X$, and we have $A_{X \times \mathbf{C}}(w)=A_{X}(v)+1$. As a result, we get

$$
L^{\mathrm{NA}}\left(\varphi_{m}\right)=\inf _{v}\left(A_{X}(v)+\varphi_{m}(v)\right)=\inf _{w}\left(A_{X \times \mathbf{C}}(w)-1-\frac{1}{m+m_{0}} w(\mathcal{J}(m \Phi))\right),
$$

and we are left showing that

$$
\inf _{w}\left(A_{X \times \mathbf{C}}(w)-w(\Phi)\right)=\lim _{m \rightarrow \infty} \inf _{w}\left(A_{X \times \mathbf{C}}(w)-\frac{1}{m+m_{0}} w(\mathcal{J}(m \Phi))\right) .
$$


But this follows formally from the inequalities

$$
w(\mathcal{J}(m \Phi)) \leq m w(\Phi) \leq w(\mathcal{J}(m \Phi))+A_{X \times \mathbf{C}}(w) .
$$

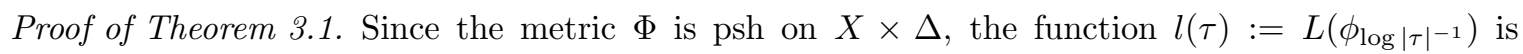
subharmonic on $\Delta$ [Bern09], and its Lelong number $\nu$ at the origin coincides with the negative of the left hand side of (3.3). We must therefore show that $\nu$ is equal to $s:=\sup _{w}\left(w(\Phi)+1-A_{X \times \mathbf{C}}(w)\right)$.

As in the proof of [Berm15, Proposition 3.8] we use that $\nu$ is the infimum of all $c \geq 0$ such that

$$
\int_{U} e^{-2(l(\tau)+(1-c) \log |\tau|)} i d \tau \wedge d \bar{\tau}=\int_{X \times U} e^{-2(\Phi+(1-c) \log |\tau|)} i d \tau \wedge d \bar{\tau}<+\infty
$$

for some small neighborhood $U \subset \Delta$ of the origin. Near each point of $X \times\{0\}$ we have $e^{-2 \Phi} i d \tau \wedge d \bar{\tau}=$ $e^{-2 \varphi} d V$, with $\varphi$ psh and $d V$ a smooth volume form. Setting $p:=\lfloor c\rfloor$ and $r=c-p \in[0,1)$ we get

$$
e^{-2(\Phi+(1-c) \log |\tau|)} i d \tau \wedge d \bar{\tau}=|\tau|^{2 p} e^{-2(\varphi+(1-r) \log |\tau|)} d V
$$

Since $\tau^{p}$ is holomorphic, it follows from [BFJ08, Theorem 5.5] that

$$
\int_{X \times U} e^{-2(\Phi+(1-c) \log |\tau|)} i d \tau \wedge d \bar{\tau}<+\infty \quad \Longrightarrow \quad \sup _{w} \frac{w(\Phi)+(1-r) w(\tau)}{p w(\tau)+A_{X \times \mathbf{C}}(w)} \leq 1,
$$

where $w$ ranges over all divisorial valuations on $X \times \mathbf{C}$. By homogeneity and by the $S^{1}$-invariance of $\Phi$, it suffices to consider $w$ that are $\mathbf{C}^{*}$-invariant and normalized by $w(\tau)=1$. We then get $w(\Phi)+1 \leq p+r+A_{X \times \mathbf{C}}(w)=c+A_{X \times \mathbf{C}}(w)$, and hence $s \leq \nu$.

Conversely, [BFJ08, Theorem 5.5] shows that

$$
\sup _{w} \frac{w(\Phi)+(1-r)}{p+A_{X \times \mathbf{C}}(w)}<1 \Longrightarrow \int_{X \times U} e^{-2(\Phi+(1-c) \log |\tau|)} i d \tau \wedge d \bar{\tau}<+\infty .
$$

Since $c$, and hence $p$ is bounded above, say by $\nu+1$, and $A_{X \times \mathbf{C}}(w) \geq 1$, the left-hand condition is equivalent to the existence of $\varepsilon>0$ such that $w(\Phi) \leq(1-\varepsilon) A_{X \times \mathbf{C}}(w)-1+c$ for all $w$.

Given $\rho>0$, we must therefore show that there exists $\varepsilon>0$ such that

$$
w(\Phi) \leq(1-\varepsilon) A_{X \times \mathbf{C}}(w)+1+s+\rho
$$

for all $w$ as above. Arguing by contradiction, we get a sequence $w_{j}$ of $\mathbf{C}^{*}$-invariant divisorial valuations with $w_{j}(\tau)=1$ such that

$$
w_{j}(\Phi) \geq\left(1-\frac{1}{j}\right) A_{X \times \mathbf{C}}\left(w_{j}\right)-1+s+\rho .
$$

Let $W$ be the subset of the Berkovich analytification $(X \times \mathbf{C})^{\text {an }}$ consisting of semivaluations $w$ that are $\mathbf{C}^{*}$-invariant and satisfy $w(\tau)=1$, see Berk90. Then $W$ is compact and even sequentially compact [Poi13], so after passing to a subsequence we may assume that $w_{j} \rightarrow w_{\infty} \in W$.

For each $m \geq 1$, the multiplier ideal sheaf $\mathcal{J}(m \Phi)$ satisfies

$$
\frac{1}{m} w_{j}(\mathcal{J}(m \Phi)) \geq w_{j}(\Phi)-\frac{1}{m} A_{X \times \mathbf{C}}\left(w_{j}\right) \geq\left(1-\frac{1}{j}-\frac{1}{m}\right) A_{X \times \mathbf{C}}\left(w_{j}\right)-1+s+\rho
$$

for all $j \geq 1$. Since $w \rightarrow w(\mathcal{J}(m \Phi))$ is continuous and $w \rightarrow A_{X \times \mathbf{C}}(w)$ is lsc on $W$, this implies

$$
\frac{1}{m} w_{\infty}(\mathcal{J}(m \Phi)) \geq\left(1-\frac{1}{m}\right) A_{X \times \mathbf{C}}\left(w_{\infty}\right)-1+s+\rho
$$

for all $m \geq 1$. In particular, $A_{X \times \mathbf{C}}\left(w_{\infty}\right)<\infty$.

On the other hand we have, by definition of $s$,

$$
\frac{1}{m} w(\mathcal{J}(m \Phi)) \leq w(\Phi) \leq A_{X \times \mathbf{C}}(w)-1+s
$$

for all divisorial valuations $w$ in $W$. By density, the inequality $\frac{1}{m} w(\mathcal{J}(m \Phi)) \leq A_{X \times \mathbf{C}}(w)-1+s$ is also valid for all quasimonomial valuations $w \in W$, and hence for all $w \in W$ by approximation via retraction to dual complexes [BFJ12, JM12]. Comparing these inequalities we infer

$$
A_{X \times \mathbf{C}}\left(w_{\infty}\right)-1+s \geq\left(1-\frac{1}{m}\right) A_{X \times \mathbf{C}}\left(w_{\infty}\right)-1+s+\rho,
$$


and we reach a contradiction by letting $m \rightarrow \infty$.

3.4. Step 4: concluding the proof of Theorem A. By 3.2 we have

$$
\lim _{m \rightarrow \infty} L^{\mathrm{NA}}\left(\varphi_{m}\right)=\lim _{t \rightarrow \infty} \frac{1}{t} L\left(\phi_{t}\right)=\lim _{t \rightarrow \infty} \frac{1}{t}\left(D\left(\phi_{t}\right)+E\left(\phi_{t}\right)\right) \leq-1+\delta^{\prime} .
$$

On the other hand, our assumption of uniform Ding stability yields

$$
L^{\mathrm{NA}}\left(\varphi_{m}\right)-E^{\mathrm{NA}}\left(\varphi_{m}\right)=D^{\mathrm{NA}}\left(\varphi_{m}\right) \geq \delta J^{\mathrm{NA}}\left(\varphi_{m}\right)=-\delta E^{\mathrm{NA}}\left(\varphi_{m}\right)
$$

and hence

$$
L^{\mathrm{NA}}\left(\varphi_{m}\right) \geq(1-\delta) E^{\mathrm{NA}}\left(\varphi_{m}\right) \geq-1+\delta
$$

by (3.1), which contradicts $\delta^{\prime}<\delta$.

3.5. The semistable case. In this section we briefly sketch the proof of Theorem B. The solvability of the twisted Kähler-Einstein equation

$$
\operatorname{Ric}(\omega)=(1-\gamma) \omega+\gamma \omega_{0}
$$

with $\gamma \in(0,1)$ is implied by the coercivity of the twisted Mabuchi K-energy

$$
M_{\gamma}:=H-(1-\gamma) E^{*}=M+\gamma E^{*} .
$$

By Theorem 2.1. K-semistability implies $D^{\mathrm{NA}} \geq 0$ on $\mathcal{H}^{\mathrm{NA}}$. Arguing as in Steps 2,3 and 4 above, we infer from this that $\lim _{t \rightarrow+\infty} D\left(\phi_{t}\right) / t \geq 0$ for each subgeodesic ray $\left(\phi_{t}\right)$ in $\mathcal{E}^{1}$. Since $M_{\gamma} \geq D+\gamma E^{*}$, this implies

$$
\lim _{t \rightarrow+\infty} \frac{M_{\gamma}\left(\phi_{t}\right)}{t} \geq \gamma \lim _{t \rightarrow+\infty} \frac{E^{*}\left(\phi_{t}\right)}{t}
$$

Since $E^{*}$ and $J$ are comparable up to multiplicative constants, we obtain an estimate

$$
\lim _{t \rightarrow+\infty} \frac{M_{\gamma}\left(\phi_{t}\right)}{t} \geq \delta \lim _{t \rightarrow+\infty} \frac{J\left(\phi_{t}\right)}{t}
$$

for some uniform constant $\delta>0$. Arguing as in Step 1 above, using the geodesic convexity of $M_{\gamma}$ established in [BB14, we deduce from this the desired coercivity estimate $M_{\gamma} \geq \delta^{\prime} J-C$, which proves the solvability of 3.5 .

\section{REFERENCES}

[Berk90] V. Berkovich. Spectral theory and analytic geometry over non-Archimedean fields. Mathematical Surveys and Monographs, vol. 33. American Mathematical Society, Providence, RI, 1990

[Berm15] R. Berman. K-polystability of Q-Fano varieties admitting Kähler-Einstein metrics. Invent. Math. Published online 23 June 2015.

[BB14] R. Berman and B. Berndtsson. Convexity of the K-energy on the space of Kähler metrics. arXiv: 1405.0401.

[BBGZ13] R. Berman, S. Boucksom, V. Guedj and A. Zeriahi. A variational approach to complex Monge-Ampère equations. Publ. Math. Inst. Hautes Études Sci. 117 (2013), 179-245.

[BBEGZ11] R. Berman, S. Boucksom, P. Eyssidieux, V. Guedj and A. Zeriahi. Kähler-Einstein metrics and the Kähler-Ricci flow on log Fano varieties. arXiv:1111.7158.

[Bern09] B. Berndtsson. Curvature of vector bundles associated to holomorphic fibrations. Ann. of Math. 169 (2009), 531-560.

[BFJ08] S. Boucksom, C. Favre and M. Jonsson. Valuations and plurisubharmonic singularities. Publ. RIMS 44 (2008), 449-494.

[BFJ12] S. Boucksom, C. Favre and M. Jonsson. Singular semipositive metrics in non-Archimedean geometry. arXiv:1201.0187. To appear in J. Algebraic Geom.

[BHJ15a] S. Boucksom, T. Hisamoto and M. Jonsson. Uniform K-stability, Duistermaat-Heckman measures and singularities of pairs. arXiv:1504.06568.

[BHJ15b] S. Boucksom, T. Hisamoto and M. Jonsson. Uniform K-stability and coercivity of the K-energy. In preparation.

[CDS15] X.X. Chen, S. K. Donaldson and S. Sun. Kähler-Einstein metrics on Fano manifolds, I-III. J. Amer. Math. Soc. 28 (2015), 183-197, 199-234, 235-278. 
[CLP14] X.X. Chen, L. Li and M. Păun. Approximation of weak geodesics and subharmonicity of Mabuchi energy. arXiv: 1409.7896.

[CSW15] X.X. Chen, S. Sun and B. Wang. Kähler-Ricci flow, Kähler-Einstein metric, and K-stability. arXiv: 1508.04397.

[Dar13] T. Darvas. Weak geodesic rays in the space of Kähler metrics and the class $\mathcal{E}\left(X, \omega_{0}\right)$. arXiv:1307.6822.

[Dar14] T. Darvas. The Mabuchi geometry of finite energy classes. arXiv:1409.2072.

[DH14] T. Darvas and W. He. Geodesic rays and Kähler-Ricci trajectories on Fano manifolds. arXiv:1411.0774.

[DR15] T. Darvas and Y. Rubinstein. Tian's properness conjectures and Finsler geometry of the space of Kähler metrics. arXiv:1506.07129v1.

[DS15] V. Datar and G. Székelyhidi. Kähler-Einstein metrics along the smooth continuity method. arXiv: 1506.07495.

[Dem92] J.-P. Demailly. Regularization of closed positive currents and Intersection Theory. J. Alg. Geom. 1 (1992) 361-409.

[DEL00] J.-P. Demailly, L. Ein, R. Lazarsfeld. A subadditivity property of multiplier ideals. Michigan Math. J. 48 (2000), 137-156.

[Der14] R. Dervan. Uniform stability of twisted constant scalar curvature Kähler metrics. arXiv:1412.0648v1.

[Fuj15] K. Fujita. Optimal bounds for the volumes of Kähler-Einstein Fano manifolds. arXiv:1508.04578.

[JM12] M. Jonsson and M. Mustaţă. Valuations and asymptotic invariants for sequences of ideals. Ann. Inst. Fourier 62 (2012), no. 6, 2145-2209.

[LX14] C. Li and C. Xu. Special test configurations and K-stability of Fano varieties. Ann. of Math. 180 (2014), 197-232.

[Li13] C. Li. Yau-Tian-Donaldson correspondence for K-semistable Fano manifolds. arXiv:1302.6681.

[PSSW08] D. H. Phong, J. Song, J. Sturm and B. Weinkove. The Moser-Trudinger inequality on Kähler-Einstein manifolds. Amer. J. Math. 130 (2008), no. 4, 1067-1085.

[Poi13] J. Poineau. Les espaces de Berkovich sont angéliques. Bull. Soc. Math. France. 141 (2013), $267-297$.

[Szé11] G. Székelyhidi. Greatest lower bounds on the Ricci curvature of Fano manifolds. Compos. Math. 147 (2011), no. 1, 319-331.

[Szé15] G. Székelyhidi. Filtrations and test-configurations. With an appendix by S. Boucksom. Math. Ann. 362 (2015), 451-484.

[Tia97] G. Tian. Kähler-Einstein metrics with positive scalar curvature. Inv. Math. 130 (1997), 239-265.

[Tia15] G. Tian. K-stability and Kähler-Einstein metrics. Comm. Pure Appl. Math. 68 (2015), 1085-1156.

Mathematical Sciences, Chalmers University of Technology and University of Gothenburg, SE-412 96 GöTEBorg, SwEdEN

E-mail address: robertb@chalmers.se

CNRS-CMls, École Polytechnique, F-91128 Palaiseau Cedex, France

E-mail address: sebastien.boucksom@polytechnique.edu

Dept of Mathematics, University of Michigan, Ann Arbor, Mi 48109-1043, USA

E-mail address: mattiasj@umich.edu 